\title{
La Cour Constitutionnelle et le droit du salarié d'ester en justice
}

\section{Melda Sur}

\section{(2) OpenEdition}

1 Journals

\section{Édition électronique}

URL : https://journals.openedition.org/rdctss/1698

DOI : 10.4000/rdctss. 1698

ISSN : 2262-9815

Éditeur

Centre de droit comparé du travail et de la sécurité sociale

\section{Édition imprimée}

Date de publication : 1 avril 2019

Pagination : 246-249

ISSN : 2117-4350

\section{Référence électronique}

Melda Sur, «La Cour Constitutionnelle et le droit du salarié d'ester en justice », Revue de droit comparé du travail et de la sécurité sociale [En ligne], 1 | 2019, mis en ligne le 01 novembre 2021, consulté le 13 novembre 2021. URL : http://journals.openedition.org/rdctss/1698; DOI : https://doi.org/10.4000/ rdctss. 1698

\section{(c) (i) (9)}

Revue de droit comparé du travail et de la sécurité sociale est mise à disposition selon les termes de la Licence Creative Commons Attribution - Pas d'Utilisation Commerciale - Pas de Modification 4.0 International. 


\section{MELDA SUR}

UNIVERSITÉ DOKUZ-EYLÜL

\section{LA COUR CONSTITUTIONNELLE ET LE DROIT DU SALARIÉ D'ESTER EN JUSTICE}

\section{I - VALIDATION DE LA LOI SUR LES TRIBUNAUX DU TRAVAIL ET DU RECOURS OBLIGATOIRE À LA MÉDIATION}

La Loi n 7036 du 12 octobre 2017 sur les Tribunaux du travail (iş Mahkemeleri Kanunu) ${ }^{1}$ avait suscité des critiques au sein de la doctrine de droit social ${ }^{2}$. Un recours pour annulation a été intenté auprès de la Cour Constitutionnelle, par 128 députés du principal parti de l'opposition (Parti républicain du peuple, Cumhuriyet Halk Partisi). La Cour Constitutionnelle dans son arrêt du 11 Juillet 2018, $n^{\circ} .2017 / 178-2018 / 82$, a statué dans le sens de la conformité de la Loi à la Constitution ${ }^{3}$.

Les principales dispositions qui avaient fait l'objet du recours étaient:

Le caractère rendu obligatoire du recours à la médiation avant l'ouverture d'une action en justice concernant toute créance et demande de réintégration fondée sur une relation de travail exceptés les cas d'accident et maladie du travail;

La soumission de la charge des frais judiciaires et d'avocat à la partie qui ne se serait pas présentée lors de la première réunion de la médiation;

Le raccourcissement des délais de prescription des créances liées à la rupture du contrat de travail (congés payés dus, indemnités de fin de contrat, indemnités de préavis, indemnité de rupture abusive et indemnité de discrimination).

A. Au sujet du premier et principal grief essentiellement fondé sur une atteinte au droit d'ester en justice garanti par l'art. 36 de la Constitution, le principal argument des requérants reposait essentiellement sur le déséquilibre de fait entre les parties. Ainsi, le travailleur qui est dans une position de faiblesse serait amené lors de la médiation à renoncer à certains de ses droits. Or la Cour Constitutionnelle établit sa décision de conformité sur plusieurs points :

La médiation reposerait sur un processus de dialogue entre les parties, pour aboutir plus rapidement et à moindres frais à une solution définitive et durable. La Cour souligne le caractère non public de ce processus qui sauvegarde ainsi la discrétion et le secret des affaires. La paix sociale serait ainsi assurée. La Cour met également en avant l'expertise exigée du médiateur, son indépendance et impartialité qui sont prévus par la législation.

1 Journal Officiel du 25 octobre 2017, $\mathrm{n}^{\circ} 30221$.

2 Voir la rubrique Turquie au n²018/1 de la Revue de Droit comparé du travail et de la sécurité sociale.

3 Arrêt publié au Journal Officiel du 11 décembre 2018, n 30622. 
La Cour mentionne également la charge excessive qui pèse sur les juridictions du travail rendant difficile une bonne administration de la justice ${ }^{4}$.

L'argument décisif nous semble être, qu'en définitive les parties, mêmes si elles sont tenues de s'adresser d'abord au médiateur, ne sont point dans l'obligation de parvenir à un accord, et finalement c'est à leur volonté qu'est liée la solution du litige. Autrement dit, le caractère obligatoire concerne uniquement le recours préalable, condition de recevabilité de l'action judiciaire. Il leur sera loisible par la suite en cas d'échec de cette phase, de recourir aux tribunaux.

Par ailleurs, la durée maximale de la médiation prévue légalement est de 3 semaines (une durée supplémentaire allant jusqu'à une semaine pourra être ajoutée en cas de nécessité, art.3/10 de la Loi sur les Tribunaux du travail) pendant laquelle les délais de forclusion et prescription seront suspendus (art. 3/17).

Enfin il est souligné que la médiation ne vient point remplacer ou entrer en concurrence avec les juridictions, et qu'elle est un procédé ayant ses propres caractéristiques venant simplement se joindre aux processus judiciaires.

B. La disposition prévoyant que les frais de médiation, ainsi que les frais judiciaires et d'avocat seraient imputés à la partie qui s'abstient de se présenter à la première réunion de médiation sans excuse valable (art. 3/12), constitue un autre point avancé par les requérants à l'encontre de la nouvelle Loi. En effet cette disposition surprend au premier abord, puisque, même la partie qui obtient gain de cause à l'issue de la procédure judiciaire, sera tenue de la totalité de ces dépenses. Une telle disposition pourrait sans doute être de nature à dissuader le travailleur d'intenter une action en vue du recouvrement de ses droits. Or la Cour souligne que seule la partie qui refuse de se présenter sans excuse valable se verrait endosser la charge des frais de justice, et que cette règle vise simplement à réduire les frais et surtout à éviter que cette phase de médiation demeure lettre morte.

Le raccourcissement des délais de prescription des créances liées à la fin du contrat de travail (congés payés dus, indemnité de fin de contrat, indemnité de préavis, indemnité de licenciement, indemnité de rupture abusive, indemnité de discrimination) qui étaient auparavant de 10 ans amenés désormais à cinq ans (art. 3 additionnel au Code du travail $n^{\circ} 4857$ ), ira certes à l'encontre des intérêts des travailleurs. Il est vrai qu'en effet c'est lorsque le travailleur se trouve délié de son lien de subordination qu'il obtient la vraie faculté d'exiger le payement de ses créances.

Toutefois, la Cour Constitutionnelle avance le motif de la nécessaire sécurité juridique des parties: en effet il est préférable de limiter la longueur des délais de forclusion, en vue d'éviter que l'une des parties se trouve pour une longue durée dans l'incertitude et sous la menace de se voir intenter une action. Le délai de prescription de 5 ans désormais prévu pour l'exigibilité des créances à partir de la fin du contrat paraît raisonnable et suffisant pour

4 Malgré les réformes entreprises, la charge qui pèse sur les chambres sociales de la Cour de cassation demeure importante: à la fin de l'année 2018, sont en attente 54108 affaires pendantes devant la $9^{\text {ème }}$ Chambre, 52716 affaires devant la 22 $2^{\text {ème }}$ Chambre, ces deux chambres s'occupant des litiges directement liés au contrat de travail et aux relations collectives. Restent 19843 affaires devant la $10^{\mathrm{ème}}$ Chambre chargée de la sécurité sociale et 15429 devant la $21^{\text {ème }}$ Chambre chargée des accidents du travail (chiffres émanant de la Présidence de la Cour de Cassation, publiés au Journal Officiel du 31 janvier 2018). 
permettre aux parties de préparer une éventuelle action en justice. Le raccourcissement de ces délais semble également plus favorable à la conservation des moyens de preuve.

\section{II - ACCROISSEMENT DES POUVOIRS PRÉSIDENTIELS DANS LE DOMAINE DES RELATIONS COLLECTIVES DE TRAVAIL}

À la suite des amendements constitutionnels intervenus par la Loi du 21.1.2017 $n^{\circ} 6771$ et mis en application après le référendum du 16 avril $2017^{5}$, le nouveau système présidentiel est mis en place, venant remplacer le séculaire système parlementaire. Désormais le Président de la République détient en sa personne le pouvoir l'exécutif et exerce les pouvoirs afférents; le Conseil des Ministres est aboli et ses pouvoirs conférés au Président de la République.

Ce changement se traduit dans le domaine des relations collectives de travail par un transfert des prérogatives des mains du Conseil des Ministres - qui, dans le nouveau système présidentiel n'existe plus - vers le Président de la République. Ainsi la procédure de l'extension des conventions collectives de travail se trouve modifiée et se fera désormais par décision présidentielle ${ }^{6}$ (art. 40 de la Loi No. 6356 sur les syndicats et des conventions collectives de travail), de même que la décision de suspension d'une grève (art. 63 de la Loi No. 6356) pour raisons de santé publique, sûreté nationale, ou affectant les transports publics et le système bancaire, qui se trouve désormais du ressort du Président de la République. Ce pouvoir de suspendre une grève est d'ailleurs usé; l'exemple le plus récent est la suspension de la grève affectant les métros à İzmir.

\section{III - LE DROIT DE RECOURS DE SALARIÉS LIMOGÉS DANS LE CADRE DES MESURES D'EXCEPTION}

Suite à la tentative de coup d'État du 15 juillet 2016, un Décret-loi n 667 a prévu des mesures d'exception à prendre dans le cadre de l'état d'urgence (Olağanüstü Hâl Kapsamında Alınan Tedbirlere Iliş̧kin Kanun Hükmünde Kararname). Parmi ces mesures, un nombre assez considérable d'employés du secteur public furent démis de leurs fonctions. Par la suite, une possibilité de recours a été prévue par le Décret-loi $n^{\circ} 685^{8}$.

Ainsi, pour ceux qui se verraient démis de leurs fonctions directement par décret, une Commission est habilitée à examiner les requêtes contre les décisions prises dans le cadre des mesures d'exception (Olağanüstü Hâl Iş̧lemlerini Inceleme Komisyonu - OHAL Komisyonu). Cette Commission a compétence pour annuler les fermetures, limogeages et licenciements directement décidés par décret, et ses sentences seront susceptibles d'appel auprès de la juridiction administrative.

La Cour Constitutionnelle a récemment rendu un arrêt intéressant (Arrêt sur requête de Ahmet Özdoğan, du 9.1.2019, $n^{\circ}$ 2017/26326) sur le droit d'ester en justice, suite au recours individuel d'un salarié employé par un sous-traitant de l'Université Gazi, et licencié

5 Journal Officiel du 27 avril 2017, $\mathrm{n}^{\circ} 30050$ bis.

6 Décret-loi (KHK 700/art. 197) du 2.7.2018.

7 Décision présidentielle $n^{\circ} 556$, Journal Officiel du 8 janvier 2019, n 30649.

8 Journal Officiel du 23 janvier 2017, n 29957. 
pour cause de lien avec l'organisation FETÖ/PDY, auteur de la tentative de coup d'Étatª . Le licenciement avait été décidé par la présidence de l'Université en vertu du décret-loi n 667 (art. 1) qui prévoit de telles mesures.

Or l'employé s'était vu refuser son recours en réintégration auprès du 21 ème Tribunal du travail d'Ankara. Le tribunal du travail avait fondé sa fin de non recevoir au motif que le requérant devait s'adresser à la Commission spécialement mandatée pour examiner les mesures d'exception. Or, cette Commission est compétente pour l'examen des mesures (incluant limogeages et licenciements) directement prises par décret et non des licenciements décidés parla hiérarchie de l'établissement, même sil'administration a agi dans le cadre des mesures d'exception. De tels licenciements, d'après la Cour constitutionnelle, ne rentreraient point dans le domaine des compétences de la Commission, et devraient demeurer du ressort des juridictions du travail.

Il existe d'ailleurs d'intéressants précédents dans la jurisprudence des chambres sociales de la Cour de Cassation:

La 22ème Chambre de la Cour de Cassation, dans son arrêt du 20 octobre 2017 ( ${ }^{\circ}$ 2017/43277 - 2017/25144), avait cassé un jugement qui avait rejeté un recours du salarié licencié dans le cadre du Décret-loi n 667. La 22 ${ }^{\mathrm{ème}}$ Chambre fonda son arrêt sur l'absence d'allégations concrètes et de preuves; elle exigea un réexamen appuyé sur des documents et renseignements qui devraient être demandés aux unités et départements compétents en matière de sûreté de l'État.

La 9ème Chambre de la Cour de Cassation, dans un arrêt du 28 mars 2018 ( n 2017/24765 - 2018/6824) a également statué dans un sens favorable au travailleur : les voies judiciaires devraient demeurer ouvertes et il appartient aux juridictions du travail de décider, au vu des preuves, s'il y a motif valable justifiant le licenciement, et en l'occurrence, un lien du requérant avec l'organisation FETÖ/PDY. Comme en l'espèce aucune preuve n'était produite ni poursuite disciplinaire introduite, la 9ème Chambre a cassé la décision de la Cour d'appel qui avait rejeté le recours en réintégration.

En définitive, la Cour Constitutionnelle, dans le cadre du recours individuel, fonde sa sentence en faveur du droit d'ester en justice garanti par l'art.6/1 de la Convention européenne des droits de l'Homme ainsi que l'art. 36 de la Constitution de la République turque. Elle souligne l'importance de ce droit qui, non seulement est un droit fondamental, mais surtout une condition de la protection effective des autres droits.

La Cour Constitutionnelle en se positionnant fait mention et tient également compte de la jurisprudence des chambres sociales de la Cour de cassation citée plus haut, pour constater qu'il y a bien eu atteinte au droit d'ester en justice et statue au renvoi aux fins de réexamen du procès ${ }^{10}$ auprès du tribunal du travail compétent.

En conclusion, la Cour Constitutionnelle, en sa jurisprudence, semble s'efforcer de maintenir un certain équilibre déjà assuré par la jurisprudence sociale dans le sens du respect du droit fondamental du salarié d'avoir accès à des tribunaux indépendants.

9 Arrêt publié au Journal Officiel du 29 Janvier 2019, n 30670.

10 Conformément à l'article 50 al. 1-2 de la Loi n 6216 du 30 mars 2011 sur l'Organisation et le fonctionnement de la Cour Constitutionnelle. 